\title{
Measurements of properties of strong and electroweak forces with the ATLAS detector at the LHC
}

\author{
Kristian Damlund Gregersen on behalf of the ATLAS \\ Collaboration $^{1}$ (D)
}

Published online: 7 February 2017

(C) The Author(s) 2017. This article is published with open access at Springerlink.com

\begin{abstract}
These proceedings summarise ATLAS measurements of properties of the strong and electroweak forces in proton-proton collisions at 7 and $8 \mathrm{TeV}$ during Run-1 at the LHC. The specific measurements presented here are concerned with the gauge symmetries in the Standard Model and related parameters, starting with the production cross section of a $W$ boson in association with jets, the forward-backward asymmetry in the production of $Z / \gamma *$ decaying to electrons or muons and extraction of the effective weak mixing angle. Then a number of measurements specifically probing electroweak processes at very high energies are presented, including the first measurement of triple gauge boson production $p p \rightarrow W(l \nu) \gamma \gamma$, the production of a leptonically decaying $W$ boson in association with $W$ or $Z$ boson decaying to jets, electroweak production of two jets in association with a $Z$ boson, and same-electric-charge diboson production in association with two jets providing the first evidence of electroweak vector boson scattering.
\end{abstract}

Keywords EWEAK $\cdot$ QCD

\section{Introduction}

The Standard Model of particle physics is built around the idea of local gauge symmetries, leading to the existence of vector bosons, mediators of the strong and electroweak forces. The production of single and multiple electroweak vector bosons in proton-proton collisions

This article is part of the Topical Collection on Proceedings of the 6th International Symposium on Symmetries in Subatomic Physics (SSP 2015), Victoria, Canada, 8-12 June 2015

Edited by Michael Gericke and Gerald Gwinner

Kristian Damlund Gregersen

k.gregersen@ucl.ac.uk

1 University College London, Gower Street, WC1E 6BT, London, UK 
in LHC Run-1 has been extensively studied by the ATLAS Collaboration [1] leading to a number of precision measurements.

The production of charged and neutral weak gauge bosons via the Drell Yan process is sensitive to higher-order effects in the strong force, the proton structure and electroweak corrections. Studying the production of a $W$ or $Z$ in association with jets provides a strong handle on these corrections. Furthermore, the interference effects between the exchange of photons and $Z$ bosons can be used for measuring Standard Model (SM) parameters with high precision, such as the weak mixing angle from the forward-backward asymmetry.

The SM gives detailed predictions on the production of multiple $W, Z$ and isolated photons, which are fixed by the gauge symmetry. Processes involving di- or triple-boson production provide important tests of the electroweak sector of the SM at high energies. Deviations from these predictions may arise from anomalous triple and quartic gauge interactions or from new particles decaying into vector bosons, which would be a hint of new physics.

The next sections summarise the individual measurements presented in this talk. The first is concerned with the strong force via the production of multiple coloured particles, while the subsequent measurements focus on different aspects of the electroweak force.

\section{Measurement of the production of a $W$ boson in association with jets}

Using data with an integrated luminosity of $4.6 \mathrm{fb}^{-1}$ collected during 2011 at a centre-ofmass energy of $7 \mathrm{TeV}$, a cross section measurement is performed for the production of a $W$ in association with up to seven jets in both the electron and muon decay channels [2]. The measurements are compared to numerous QCD predictions including next-to-leadingorder perturbative calculations for up to five jets, resummation calculations and Monte Carlo generators spanning five orders of magnitude in the production cross section. Jets with transverse momenta up to $1 \mathrm{TeV}$ are observed, probing a kinematic regime where higher-order electroweak effects can become as important as those from higher order perturbative QCD corrections.

Figure 1 shows the measured cross sections for different jet multiplicities and transverse momenta of the leading jet $p_{\mathrm{T}}$. The observed decrease in cross section when going to higher multiplicities, and in particular the ratio between two adjacent jet bins, confirms the socalled stair case scaling which is closely linked to the non-Abelian nature of the gauge group describing the strong force [3].

The theoretical predictions are in general in good agreement with the data. However, there is currently no theoretical prediction that is able to provide an accurate description of the data for all measured differential cross sections. The data presented in this analysis will allow a better quantitative understanding of perturbative QCD, as well as future comparisons to predictions which include electroweak corrections.

\section{Forward-backward asymmetry and the weak mixing angle}

This analysis [6] presents measurements of the forward-backward asymmetry in the reaction $p p \rightarrow Z / \gamma * \rightarrow l^{+} l^{-}$, with $l$ being electrons or muons, and the extraction of the effective weak mixing angle. The results are based on the full set of data collected in 2011 at a centre-of-mass energy of $7 \mathrm{TeV}$, corresponding to an integrated luminosity of $4.8 \mathrm{fb}^{-1}$. 


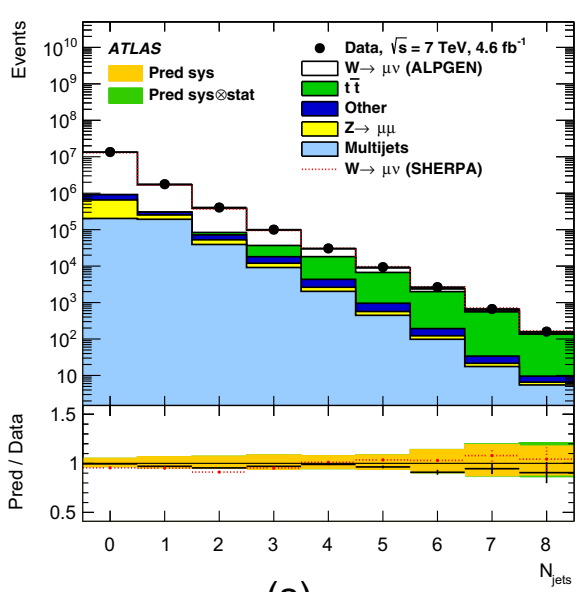

(a)

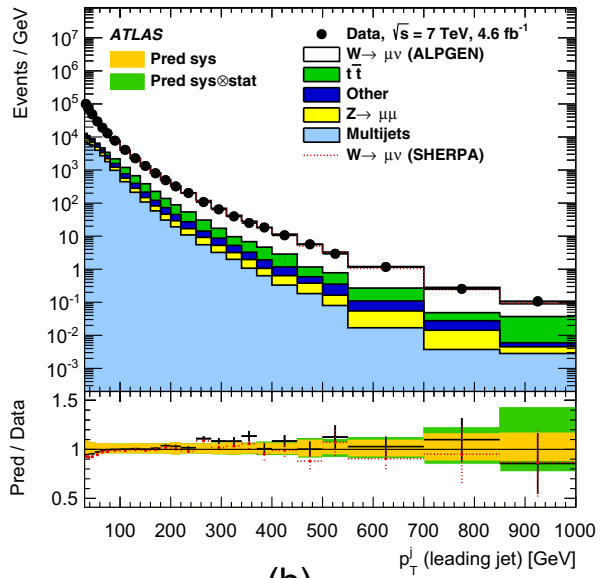

(b)

Fig. 1 Distribution of events passing the $W+$ jets selection as a function of (a) the inclusive jet multiplicity, $N_{\text {jets }}$, and (b) the leading jet $p_{\mathrm{T}}$ for the muon channel. On the data points, the statistical uncertainties are smaller than the size of the points. The lower panels show ratios of the predictions for signal and background to the data, where ALPGEN [4] (black line) and SHERPA [5] (red dashed line) is used for the signal simulation, [2]

The production cross section for the neutral current annihilation process is given by [6]

$$
\frac{\mathrm{d} \sigma}{\mathrm{d}(\cos \theta)}=\frac{4 \pi \alpha^{2}}{3 \hat{s}}\left[\frac{3}{8} A\left(1+\cos ^{2} \theta\right)+B \cos \theta\right]
$$

where $\alpha$ is the fine-structure constant, $\sqrt{\hat{s}}$ is the centre-of-mass energy of the quark and anti-quark, and $\theta$ is the angle between the negatively charged lepton and the quark in the rest frame of the dilepton system. The coefficients $A$ and $B$ are functions of $\sqrt{\hat{s}}$ and the electroweak quantum numbers of the quarks and leptons involved in the process as well as the weak mixing angle.

When the dilepton system has non-vanishing transverse momentum, the four-momentum of the incoming (anti-)quark is not known, as it is no longer collinear with the incoming beams. The impact of this effect on the asymmetry measurement is minimised by choosing a particular rest frame of the dilepton system, the Collins-Soper (CS) frame [7], in which the angle between the lepton and the quark, $\theta_{\mathrm{CS}}^{*}$, is defined with respect to the direction of the quark.

The forward-backward asymmetry, $A_{\mathrm{FB}}$, is then defined as

$$
A_{\mathrm{FB}}=\frac{N_{\cos \theta_{\mathrm{CS}}^{*} \geq 0}-N_{\cos \theta_{\mathrm{CS}}^{*}<0}}{N_{\cos \theta_{\mathrm{CS}}^{*} \geq 0}+N_{\cos \theta_{\mathrm{CS}}^{*}<0}^{*}}=\frac{3 B}{8 A} .
$$

The analysis divides the electron pairs into two distinct categories: $\mathrm{CC}$ and $\mathrm{CF}$, where $\mathrm{C}$ is central and $\mathrm{F}$ is forward in terms of pseudorapidity, $\eta$, the difference being that the forward electrons have no tracking information. Figure 2a shows the measurement of $A_{\mathrm{FB}}$ as function of the invariant mass of the electron pair. The measured asymmetry values are found to be in agreement with the corresponding Standard Model predictions. 


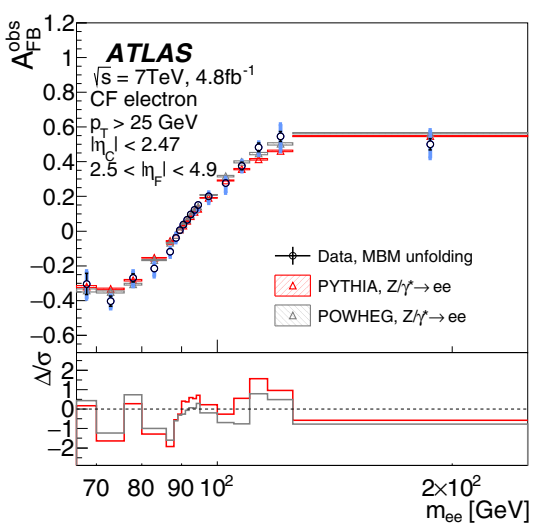

(a)

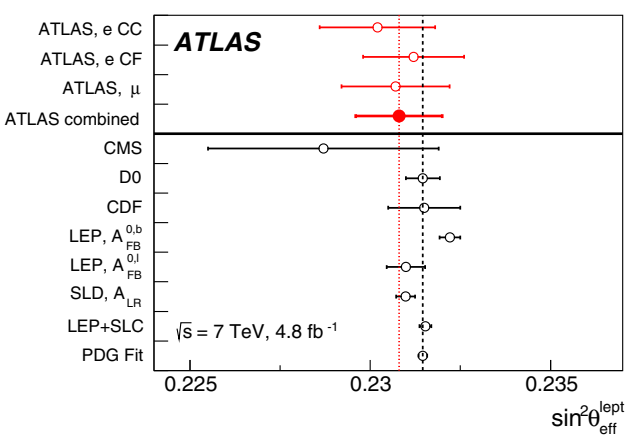

(b)

Fig. 2 a Forward-backward asymmetry values as a function of the dilepton invariant mass for the CF electron channel compared to truth-level MC information [6]. The lower panel shows the pull values. b Comparison of the results of this analysis with other published results for $\sin ^{2} \theta_{\text {eff }}^{\text {lept }}[8,9]$, and [10]. The vertical dotted line shows the central value of the ATLAS combined measurement reported here, while the vertical dashed line represents that of the current PDG global fit [11]

The forward-backward asymmetry in lepton pair production is directly related to one of the fundamental parameters in the electroweak theory - the weak mixing angle. In terms of the underlying gauge symmetry, the weak mixing angle is the angle by which the spontaneous symmetry breaking rotates the plane of the original mass-less gauge bosons to produce the physical gauge bosons. It also relates the masses of the $W$ and $Z$ bosons. The value of the weak mixing angle is not predicted by the SM, and there is no generally accepted theory that explains its value.

The weak mixing angle is extracted from the $A_{\mathrm{FB}}$ measurement by creating templates with different values of the weak mixing angle and performing a $\chi^{2}$ test to find the best match. The mass of the $Z$ boson is kept fixed in the process.

The combination of the muon and electron channels yields a value for the effective weak mixing angle of

$$
\left.\left.\sin ^{2} \theta_{\text {eff }}^{\text {lept }}=0.2308 \pm 0.0005 \text { (stat. }\right) \pm 0.0006 \text { (syst. }\right) \pm 0.0009(\mathrm{PDF}),
$$

where the first uncertainty corresponds to data statistics, the second to systematic effects and the third to knowledge of the parton density functions. As seen in Fig. 2, this result agrees with the current world average.

\section{Semileptonic $W W+W Z(\rightarrow l v j j)$ and anomalous triple gauge couplings}

The production of a $\mathrm{W}$ boson decaying to $e v$ or $\mu \nu$ in association with a $W$ or $Z$ boson decaying to two jets is studied using $4.6 \mathrm{fb}^{-1}$ of proton-proton collision data at a centre-ofmass energy of $7 \mathrm{TeV}[12]$.

The cross sections for $W W$ and $W Z$ production at the LHC have previously been measured in ATLAS in the fully leptonic final states [14, 15]. The semileptonic final states suffer from larger backgrounds from $W$ or $Z$ boson production in association with jets, but 


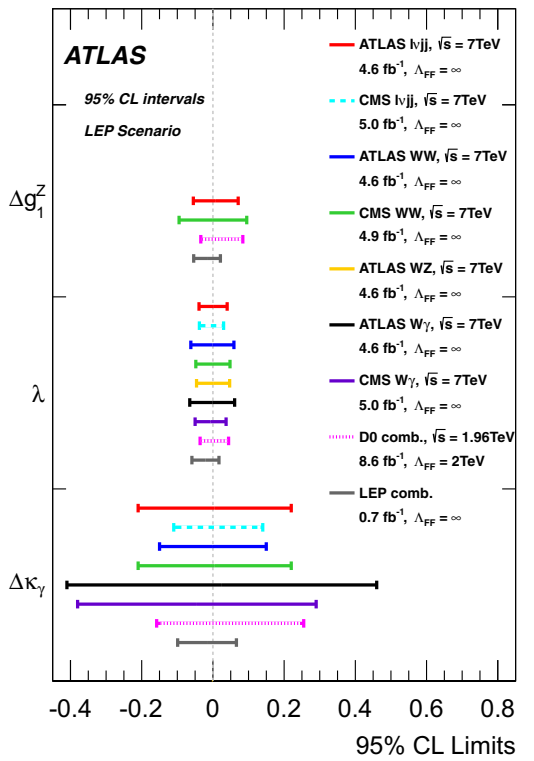

(a)

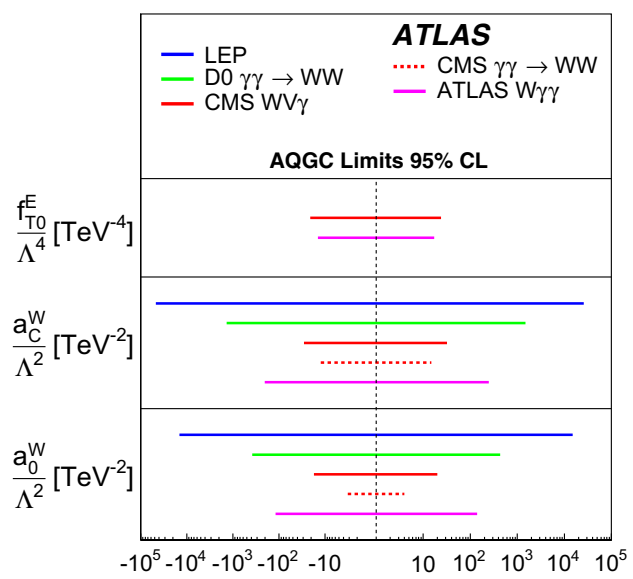

(b)

Fig. 3 Comparison of limits on anomalous triple (a) and quartic (b) gauge coupling parameters obtained in this analysis with limits quoted by other experiments and/or in different channels, [12] and [13]

benefit from significantly larger branching fractions than the fully leptonic states and thus represent important complementary measurements.

The combined $W W+W Z$ cross section in this analysis is measured with a significance of $3.4 \sigma$ and is found to be $68 \pm 7$ (stat.) \pm 19 (syst.) pb, in agreement with the Standard Model expectation of $61.1 \pm 2.2 \mathrm{pb}$ as predicted by MC@NLO [16].

The triple gauge couplings describing the $W W Z$ and $W W \gamma$ vertices are given as five dimensionless parameters: $\lambda_{\gamma}, \lambda_{Z}, \kappa_{\gamma}, \kappa_{Z}$, and $g_{1}^{Z}$, only considering couplings that conserve $C$ and $P$ and satisfy electromagnetic gauge invariance [17]. In the SM, $\lambda_{V}=0, \kappa_{V}=1$ and $g_{1}^{Z}=1$. Various assumptions can be made to decrease the number of free parameters; here, the so-called LEP scenario [18] is used, in which $\lambda_{\gamma}=\lambda_{Z}$ and $\Delta \kappa_{Z}=\Delta g_{1}^{Z}-\Delta \kappa_{\gamma} \tan ^{2} \theta_{W}$ where $\Delta \kappa_{V} \equiv \kappa_{V}-1, \Delta g_{1}^{Z} \equiv g_{1}^{Z}-1$ and $\theta_{W}$ is the weak mixing angle. This results in three free parameters: $\lambda, \Delta \kappa_{\gamma}$ and $\Delta g_{1}^{Z}$.

The observable effect of non-zero anomalous couplings is an enhancement of the differential cross section at high scattering energies. Limits on the anomalous couplings are set by fitting the distribution of the transverse momentum of the dijet system. Figure 3a shows the derived limits on $\lambda, \Delta \kappa_{\gamma}$ and $\Delta g_{1}^{Z}$ compared to previous measurements and are all consistent with zero, in agreement with the predictions of the SM.

\section{$5 W \gamma \gamma$ production and anomalous quartic gauge couplings}

This analysis [19] reports evidence of triple gauge boson production $p p \rightarrow W(l v) \gamma \gamma+X$, which is accessible for the first time with the $8 \mathrm{TeV}$ LHC data set. The fiducial cross section for this process is measured in a data sample corresponding to an integrated luminosity of 


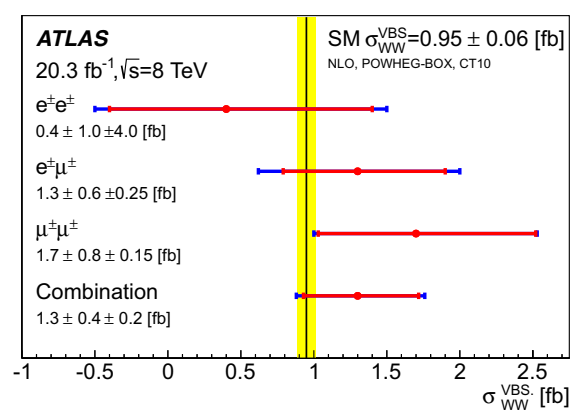

(a)

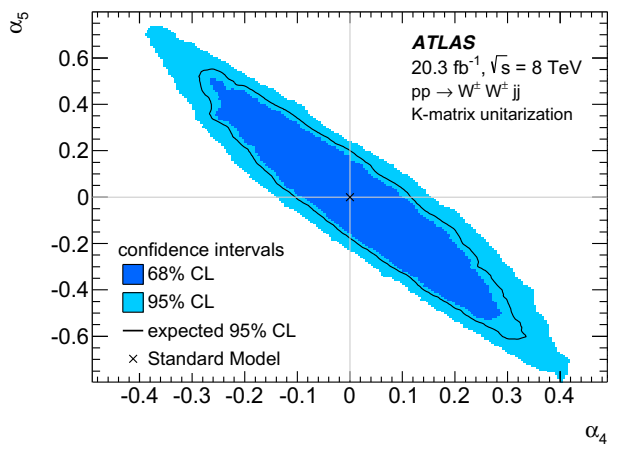

(b)

Fig. 4 a Measured cross section for the vector boson scattering process, [26]. b Limits on $\left(\alpha_{4}, \alpha_{5}\right)$ at $95 \%$ and $68 \%$ confidence levels, [23]

$20.3 \mathrm{fb}^{-1}$, collected by the ATLAS detector in 2012. Events are selected using the $W$ boson decay to $e v$ or $\mu \nu$ as well as requiring two isolated photons.

The final states under consideration mainly come from events where the $W$ boson is produced in the hard interaction between the two partons, and the photons either originate from initial or final state radiation processes, or from triple or quartic gauge vertices together with the $W$ boson.

Deviations from the SM prediction of the quartic gauge vertex are described in terms of anomalous quartic gauge couplings (aQGCs). The aQGCs are introduced as dimension- 8 operators following the formalism defined in Ref. [20]. While many operators give rise to anomalous couplings of the form $W W \gamma \gamma$, this study is restricted to $f_{\mathrm{T} 0} / \Lambda^{4}, f_{\mathrm{M} 2} / \Lambda^{4}$, and $f_{M} 3 / \Lambda^{4}$, where $\Lambda$ represents the scale at which new physics appears, and $f$ the coupling of the respective operator. The $W \gamma \gamma$ final state is expected to be particularly sensitive to the T0 operator. To preserve unitarity up to high energy scales, a form factor is introduced which depends on the energy, the form factor scale $\Lambda_{\mathrm{FF}}$ and an exponent $n$ following [21] and [22]. Figure $3 \mathrm{~b}$ shows the derived limits on the aQGCs which agree with the SM predictions.

\section{Vector boson scattering}

This analysis [23] presents the first study of $W^{ \pm} W^{ \pm} j j$, same-electric-charge diboson production in association with two jets, using $20.3 \mathrm{fb}^{-1}$ of proton-proton collision data at a centre-of-mass energy of $8 \mathrm{TeV}$.

Two classes of processes give rise to this final state. The first process, which includes contributions from scattering of two vector bosons, involves exclusively weak vertices and is referred to as electroweak production. The second process involves both the strong and electroweak vertices and is referred to as strong production. In the case of sameelectric-charge $W W$ production, the strong production cross section does not dominate the electroweak cross section, making this channel an ideal choice for initial studies on vector boson scattering.

In order to isolate the vector boson scattering process from the background coming from strongly produced $W W$ with jets, a cut is placed on the angular separation between the 


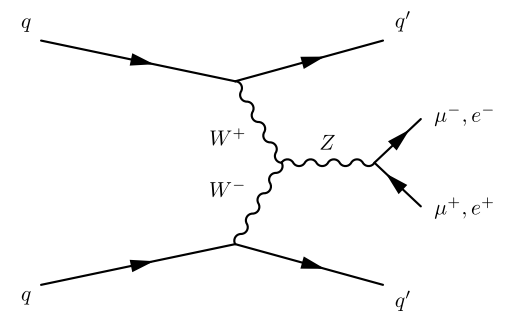

(a)

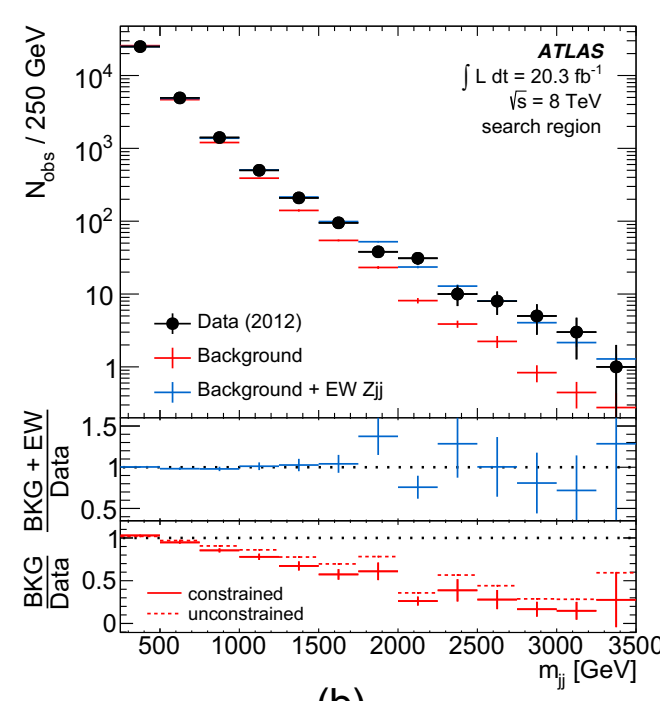

(b)

Fig. 5 a Feynman diagram for production of a $Z$ boson in association with jets via electroweak vector boson fusion. b The dijet invariant mass distribution, [27]. The lowest panel shows the ratio of constrained and unconstrained background templates to the data

two jets. The strongly produced process will have colour connected quarks in the final state which tend to be closer together than the forward jets in the vector boson scattering process.

The vector boson scattering process is a key process to probe the nature of electroweak symmetry breaking. Without the interference from the SM Higgs boson, and potentially also new physics, it violates unitarity of the scattering matrix at energies around $1 \mathrm{TeV}$. It is also sensitive to non-SM contributions to the quartic gauge boson vertex $W W W W$, which can be parameterised in terms of two parameters, $\left(\alpha_{4}, \alpha_{5}\right)[24,25]$, which are zero in the SM.

First evidence for $W^{ \pm} W^{ \pm} j j$ production and electroweak-only $W^{ \pm} W^{ \pm} j j$ production is observed with a significance of 4.5 and 3.6 standard deviations, respectively. The measured production cross sections are in agreement with Standard Model predictions and limits at $95 \%$ confidence level are set on anomalous quartic gauge Fig. $4 a$ and b.

\section{Vector boson fusion with $Z$ boson}

Measurements of fiducial cross sections for the electroweak production of two jets in association with a $Z$ boson are performed using $20.3 \mathrm{fb}^{-1}$ at a centre-of-mass energy of $8 \mathrm{TeV}$ [27].

Electroweak $Z j j$ production in the leptonic decay channel is defined as all contributions to $l^{+} l^{-} j j$ production for which there is a $t$-channel exchange of an electroweak gauge boson. This includes $Z$ boson production via vector boson fusion (VBF) shown in Fig. 5a, $Z$ boson bremsstrahlung and non-resonant production. The VBF process is of particular interest because of the similarity to the VBF production of a Higgs boson and the sensitivity to anomalous $W W Z$ triple gauge couplings.

The electroweak component is extracted by a fit to the dijet invariant mass distribution in a fiducial region chosen to enhance the electroweak contribution over the dominant 
background in which the jets are produced via the strong interaction. The electroweak cross sections measured in two fiducial regions are in good agreement with the Standard Model expectations and the background-only hypothesis is rejected with significance above the $5 \sigma$ level (see Fig. 5b). Furthermore, limits are derived for the anomalous triple gauge couplings for the $W W Z$ vertex.

\section{Conclusion}

ATLAS measurements of properties of the strong and electroweak forces at centre-of-mass energies of 7 and $8 \mathrm{TeV}$ during Run-1 of the LHC are presented here. The production cross sections of the measured processes span several orders in magnitude and probe previously uncharted territory. The results are in general in good agreement with the predictions from the SM.

Open Access This article is distributed under the terms of the Creative Commons Attribution 4.0 International License (http://creativecommons.org/licenses/by/4.0/), which permits unrestricted use, distribution, and reproduction in any medium, provided you give appropriate credit to the original author(s) and the source, provide a link to the Creative Commons license, and indicate if changes were made.

\section{References}

1. ATLAS Collaboration: JINST 3, S08003 (2008)

2. ATLAS Collaboration: Eur. Phys. J. C 75, 82 (2015)

3. Englert, C., Plehn, T., Schichtel, P., Schumann, S.: Phys, Rev. D 83 (2011). arXiv:1102.4615 [hep-ph]

4. Mangano, M.L., et al.: J. High Energy Phys. 0307, 001 (2003). arXiv:hep-ph/0206293 [hep-ph]

5. Gleisberg, T., et al.: J. High Energy Phys. 0902, 007 (2009). arXiv:0811.4622 [hep-ph]

6. ATLAS Collaboration: JHEP 09, 049 (2015). arXiv:1503.03709 [hep-ex]

7. Collins, J.C., Soper, D.E.: Phys. Rev. D 16, 2219 (1977)

8. CMS Collaboration: Phys. Rev. D 84, 112002 (2011). arXiv:1110.2682 [hep-ex]

9. Abazov, V.M., et al.: (D0 Collaboration), arXiv:1408.5016 [hep-ex]

10. Altonen, T.A., et al.: CDF and D0 Collaborations. Phys. Rev. D 89, 072005 (2014). arXiv:1402.2239 [hep-ex]

11. Nakamura, K., et al.: Particle Data Group. Rev. Part. Phys., J. Phys. G 37, 075021 (2010)

12. ATLAS Collaboration: JHEP 01, 049 (2015). arXiv:1410.7238 [hep-ex]

13. ATLAS Collaboration: Phys. Rev. Lett. 115, 031802 (2015). arXiv:1503.03243 [hep-ex]

14. ATLAS Collaboration: Phys. Rev. D 87, 112001 (2013). arXiv:1210.2979 [hep-ex]

15. ATLAS Collaboration: Eur. Phys. J. C 72, 2173 (2012). arXiv:1208.1390 [hep-ex]

16. Frixione, S., Webber, B.R.: JHEP 06, 029 (2002). arXiv:1102.4615 [hep-ph]

17. Hagiwara, K., Peccei, R., Zeppenfeld, D., Hikasa, K.: Nucl. Phys. B 282, 253 (1987)

18. Altarelli, G., Sjötrand, T., Zwirner, F.: Physics at LEP2 CERN (1996)

19. ATLAS Collaboration: Phys. Rev. Lett. 115, 031802 (2015). arXiv:1503.03243 [hep-ex]

20. Eboli, O.J.P., Gonzalez-Garcia, M.C., Mizukoshi, J.K.: Phys. Rev. D 74, 073005 (2006). arXiv:hep-ph/0606118

21. Baur, U., Zeppenfeld, D.: Phys. Lett. B 201, 383 (1988)

22. Aihara, H., et al.: arXiv:hep-ph/9503425

23. ATLAS Collaboration: Phys. Rev. Lett. 113, 141803 (2014). arXiv:1405.6241 [hep-ex]

24. Alboteanu, A., Kilian, W., Reuter, J.: J. High Energy Phys. 11, 010 (2008)

25. Appelquist, T., Bernard, C.: Phys. Rev. D 22, 200 (1980). Longhitano, A. C.: Phys. Rev. D 22, 1166 (1980); Nucl. Phys. B 188, 118 (1981)

26. ATLAS Collaboration: Phys. Rev. Lett. 113, 141803 (2014). arXiv:1405.6241 [hep-ex]

27. ATLAS Collaboration: JHEP 04, 031 (2014). arXiv:1401.7610 [hep-ex] 